\title{
Dynamic Gain Control of Dopamine Delivery in Freely Moving Animals
}

\author{
P. Read Montague, ${ }^{1}$ Samuel M. McClure, ${ }^{1}$ P. R. Baldwin, ${ }^{1}$ Paul E. M. Phillips,${ }^{2,3,4}$ Evgeny A. Budygin, ${ }^{3,4}$ \\ Garret D. Stuber, ${ }^{2,3,4}$ Michaux R. Kilpatrick, ${ }^{3,4}$ and R. Mark Wightman ${ }^{3,4}$ \\ ${ }^{1}$ Human Neuroimaging Laboratory, Center for Theoretical Neuroscience, Division of Neuroscience, Baylor College of Medicine, Houston, Texas 77030, and \\ Departments of ${ }^{2}$ Psychology, ${ }^{3}$ Chemistry, and ${ }^{4}$ Neuroscience Center, University of North Carolina at Chapel Hill, Chapel Hill, North Carolina 27599
}

Activity changes in a large subset of midbrain dopamine neurons fulfill numerous assumptions of learning theory by encoding a prediction error between actual and predicted reward. This computational interpretation of dopaminergic spike activity invites the important question of how changes in spike rate are translated into changes in dopamine delivery at target neural structures. Using electrochemical detection of rapid dopamine release in the striatum of freely moving rats, we established that a single dynamic model can capture all the measured fluctuations in dopamine delivery. This model revealed three independent short-term adaptive processes acting to control dopamine release. These short-term components generalized well across animals and stimulation patterns and were preserved under anesthesia. The model has implications for the dynamic filtering interposed between changes in spike production and forebrain dopamine release.

Key words: dopamine; plasticity; facilitation; depression; reward; voltammetry

\section{Introduction}

During reward-based learning tasks in alert primates, single-unit recordings in midbrain dopamine neurons demonstrate that phasic activity encodes the ongoing difference between experienced reward and expected reward (prediction error) that complies with the basic assumptions of learning theory (Montague and Sejnowski, 1994; Montague et al., 1996; Schultz et al., 1997; Hollerman and Schultz, 1998; Schultz and Dickinson, 2000; Waelti et al., 2001). However, these electrophysiological data provide no information on the transformation from spikeencoded reward-prediction errors to dopamine delivery that modulates reward-seeking behavior (Phillips et al., 2003b). To bridge this gap, we made rapid electrochemical dopamine measurements in the striatum of freely moving rats while delivering multiple patterns of electrical stimulation to dopamine axons. This revealed rich dynamic adaptation of dopamine release that attends changes in spike production.

Previous work measuring dopamine release in anesthetized rats has demonstrated that after brief, intense stimulation of dopamine neurons, $\sim 20 \mathrm{~min}$ is required for complete recovery of dopamine release (Michael et al., 1987). These findings inspired a

Received July 8, 2003; revised Nov. 18, 2003; accepted Dec. 23, 2003.

This work was supported by the National Institute on Drug Abuse, National Institute of Mental Health, and Kane Family Foundation. We thank Drs. Regina Carelli, Peter Dayan, Ron Fisher, Margaret Rice, Donita Robinson, and Michael Wiest for constructive comments and criticisms.

Correspondence should be addressed to either of the following: P. Read Montague (model), Human Neuroimaging Laboratory, Center for Theoretical Neuroscience, Division of Neuroscience, Baylor College of Medicine, 1 Baylor Plaza, Houston, TX77030, E-mail: read@bcm.tmc.edu; or Paul E. M. Phillips (experimental), Department of Psychology, Davie Hall CB 3270, University of North Carolina, Chapel Hill, NC 27599, E-mail: pemp@unc.edu.

S. M. McClure's present address: Department of Psychology, Princeton University, Princeton, NJ 08540.

DOI:10.1523/JNEUROSCI.4279-03.2004

Copyright $\odot 2004$ Society for Neuroscience $\quad$ 0270-6474/04/241754-06\$15.00/0 fixed amplitude model of dopamine release (Wightman et al., 1988) that was used to fit dopamine release data provided that stimulation frequencies remained extremely low. However, more recent work using less intense, but more rapidly repeated electrical stimuli, shows that rich, dynamic fluctuations occur in response to intracranial self-stimulation (Garris et al., 1999; Yavich and Tiihonen, 2000) or experimenter-delivered stimuli in vivo (Kilpatrick et al., 2000; Yavich and MacDonald, 2000) and in vitro (Cragg, 2003). Collectively, these observations suggested the hypothesis that multiple dynamic components modulate dopamine delivery, thus contradicting fixed-amplitude models of release (Wightman et al., 1988). We show here that a single dynamic model captures all the measured changes in ongoing dopamine release, provides evidence of a family of short-term adaptation mechanisms in dopamine release, and provides further insight into the role of this important neuromodulatory system.

\section{Materials and Methods}

In vivo measurements. Striatal dopamine was measured in rats using methods described previously (Garris et al., 1997; Phillips et al., 2003a). Animal care was approved by the Institutional Animal Care and Use Committee of the University of North Carolina. Stereotaxic surgery was performed to chronically implant (1) a bipolar stimulating electrode in the substantia nigra/ventral tegmental area, (2) a guide cannula above the ipsilateral caudate-putamen, and (3) an $\mathrm{Ag}-\mathrm{AgCl}$ reference electrode. After full recovery, experiments were performed by lowering a carbonfiber microelectrode through the guide cannula into a position in the caudate-putamen where dopamine release was optimized. Extracellular dopamine was measured by fast-scan cyclic voltammetry $(-0.4$ to +1.0 $\mathrm{V}$ to $-0.4 \mathrm{vs} \mathrm{Ag} / \mathrm{AgCl}, 300 \mathrm{~V} / \mathrm{sec}$ ) every $100 \mathrm{msec}$ during patterns of electrical stimulation. Carbon-fiber microelectrodes were calibrated in vitro after use with a dopamine stock solution. In some experiments, 
measurements were made under urethane anesthesia (1.5 gm $/ \mathrm{kg}$; Wightman et al., 1988).

Formulation of the model. A previous model of dopamine delivery (Wightman et al., 1988) sets the rate of change of dopamine concentration $(C)$ equal to: $(d C / d t)=$ rate added through release - rate removed by uptake.

In its simplest form, a fixed concentration of dopamine $C_{p}$ is released with each stimulus pulse, and uptake follows Michaelis-Menten kinetics characterized by a maximal velocity $V_{m}$ and affinity constant, $K_{m}$. This can be formulated as:

$$
\frac{d C}{d t}=r C_{p}-V_{m}\left(1+K_{m} / C\right)^{-1}
$$

where $r$ represents the impulse rate. However, at normal physiological impulse rates, the complex dynamics apparent in the experimental data cannot be captured with this simple approach (see variability of responses in Fig. $1 B$ ), and a more sophisticated approach is required.

The parameters for dopamine reuptake $\left(V_{\mathrm{m}}\right.$ and $K_{\mathrm{m}}$ ) have been shown to be fairly stable (Venton et al., 2003), consequently, we focused on the limitations implicit in the first term $r C_{p}$, which characterizes dopamine release according to the average impulse rate $r$ and the average release per impulse $C_{p}$. Deviating from this approach, we modeled the concentration of dopamine added by each impulse as a product of two time-dependent functions $p(t) A(t) . A(t)$ is a function that depends on independent facilitation and depression components $I_{j}(t)$, and its initial value, $a_{0} \cdot p(t)$ is a function that models the exact impulse times for a specific pattern of impulses. As detailed below, each $I_{j}(t)$ possesses "kick and relax" dynamics.

In this new approach, as with the previous fixed amplitude model, the Michaelis-Menten variables for uptake $\left(V_{\mathrm{m}}\right.$ and $\left.K_{\mathrm{m}}\right)$ were not used as free parameters in the model, but were instead maintained at empirically determined constant values of $4.0 \mu \mathrm{M} / \mathrm{sec}$ and $0.2 \mu \mathrm{M}$, respectively (Wightman and Zimmerman, 1990). The dynamic gain control of release is captured by the time varying function $A(t)$; itself composed of separate "hidden" dynamic processes $I_{j}$. This approach is analogous to that of Abbott et al. (1997) and Varela et al. (1997), addressing short-term changes in glutamatergic transmission, except that all factors are multiplicative.

$$
A(t)=a_{0} \prod_{j=1}^{N} I_{j}(t)
$$

The kick and relax dynamics are straightforward. At each spike, $I_{j}$ is multiplied (kicked) by a factor $k_{j}$, so that, $I_{j}$ is replaced by $k_{j} I_{j}\left(k_{j}>1\right.$ gives facilitation, whereas $k_{j}<1$ gives depression). During interstimulus periods, the independent dynamic factors decay to their equilibrium value of 1.0 with first order kinetics (time constant $\tau_{j}$ ) so that their product $A(t)$ decays back to its initial value $a_{0}$ :

$$
\frac{d I_{j}}{d t}=\tau_{j}^{-1}\left(1-I_{j}\right)
$$

As indicated above, the fixed rate $r$ is replaced by a function $p(t)$ that describes the entire pattern of impulses evoked in the dopamine neurons by the stimulating electrode. With these changes, Equation 1 becomes:

$$
\frac{d C}{d t}=p A-V_{m}\left(1+K_{m} / C\right)^{-1}
$$

introduction of receptive female

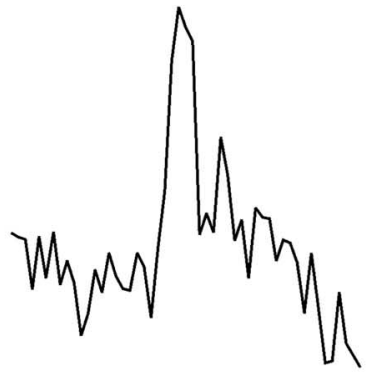

Figure 1. Extracellular dopamine in the caudate-putamen of freely moving rats. $A, A$ dopamine transient evoked by a repetitive electrical stimulation (24 pulses, $60 \mathrm{~Hz}, 120 \mu \mathrm{A}$; horizontal bar) delivered to dopamine neurons in the substantia nigra/ 政 Dopamine concentration transients evoked by an irregular stimulation train where each stimulus (24 pulses; vertical bar) is a repetitive electrical stimulation identical to that delivered in $A$. The irregular stimulus pattern is a "playback" of an intracranial self-stimulation lever-press pattern of another rat. Both facilitation (compare amplitudes at 1 and 2) and depression (3) in the dynamics governing evoked release are apparent.

Data fitting. A direct integration approach was used to estimate the model $(C)$ based on the measured dopamine fluctuations. The stimulation pattern $p(t)$ was modeled explicitly as $p(t)=\sum \epsilon\left(t-t_{i}\right)$ where $\epsilon(t)=\tau_{R}{ }^{-1} \exp \left\{-\left(t-t_{i}\right) / \tau_{R}\right\}$. The free parameters in this approach were the kick values $k_{j}$, the associated time constants $\tau_{j}$, the initial value $a_{O}$, and the time constant of the electrochemical probe $\tau_{R}$. The time constant $\tau_{R}$ accounts for the delays due to diffusion in the extracellular space and the response time of the electrode. That is, $\tau_{R}$ characterizes the low pass filtering that occurs with this type of electrochemical measurement (Bath et al., 2000). For each fit of the model to the measured dopamine levels, the number of dynamic components $I_{j}$ and their polarity (facilitating or depressing) were preselected. The optimization of the fits used Powell's method with a fourth order integration scheme (Powell, 1964; Press et al., 1990). The fits produced the greatest error reduction for one facilitating factor and two depression components, and no substantial improvement for four or more components. In all cases, a large range of parameter values was explored by the fitting procedure. Furthermore, this direct integration approach yielded fitted values for the electrode time constant $\tau_{R}$ in the range of previous empirical measurements of its value $(\sim 200$ msec; Bath et al., 2000). In this direct integration approach, $C$ was computed first followed by updating of the dynamic components $I_{j}$. 


\section{Results}

Striatal dopamine release was evoked by electrical stimulation of dopaminergic cell bodies and monitored with fast-scan cyclic voltammetry at carbon-fiber microelectrodes. In freely moving rats, both regular and irregular temporal patterns of repetitive stimulation were used to elicit dopamine release. The neurochemical response to a single stimulus train was similar to dopamine release evoked by salient behavioral cues (Fig. 1A) (see also Robinson et al., 2002). The irregular patterns of stimulation were the recorded lever-press records of other animals during selfstimulation experiments (patterns taken from self-stimulation experiments described in Kilpatrick et al., 2000). As shown in Figure $1 B$, the richness of the dynamic influences on dopamine release is apparent for an irregular stimulation pattern in which both facilitation (arrow 2) and depression (arrow 3 ) of release, compared with the initial event (arrow 1), are evident. This depression is reversible, demonstrated by a significant recovery of release after $15 \mathrm{~min}$ (data not shown). Dynamic changes also emerge using regular interval patterns of stimulation (Fig. 2D-F).

The model described in Materials and Methods was fit to these experimental data to determine values for the kick, $k_{j}$, and the time constant, $\tau_{j}$, for each independent dynamic component $I_{j}$, and $a_{0}$, the initial estimate of dopamine released per stimulus pulse (Table 1). Error analysis was used to select the number of unique dynamic factors in any particular fit. Three key features emerged from this error analysis. (1) We find that there is a dramatic reduction in residual error as the number of independent components ( $I$ values) is increased from 1 to 3 , and very little reduction thereafter (Fig. 2A). (2) The observed dopamine dynamics are consistently captured by one facilitating $\left(k_{j}>1\right)$, and two depressing $\left(k_{j}<1\right)$ components (Fig. $2 B$ ). (3) The number and type of the dynamic components does not change with anesthesia.

The model captures the extracellular dopamine dynamics for an individual repetitive stimulus (Fig. 2C) as well as interstimulus dynamics during regular patterned stimulation in both anesthetized

(Fig. 2D) and freely moving animals (Fig. 2 E,F). In alert animals, regular patterns of repetitive stimuli generated dopamine release profiles that revealed individual facilitation and depression suggested by the rich dopamine dynamics observed in Figure $1 B$. When stimulation trains are repeated every $2 \mathrm{sec}$, the first $\sim 10$ trains elicit enhanced dopamine (Fig. $2 E$ ), whereas with $5 \mathrm{sec}$ intervals there is decremented release (Fig. $2 F)$. Good fits $\left(r^{2}=\right.$

A

C

E
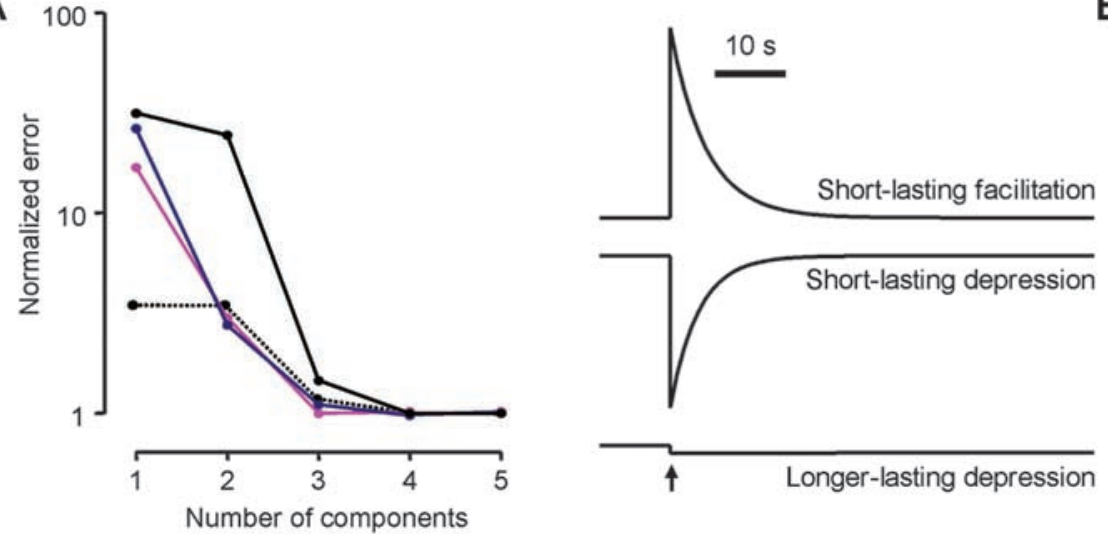

B
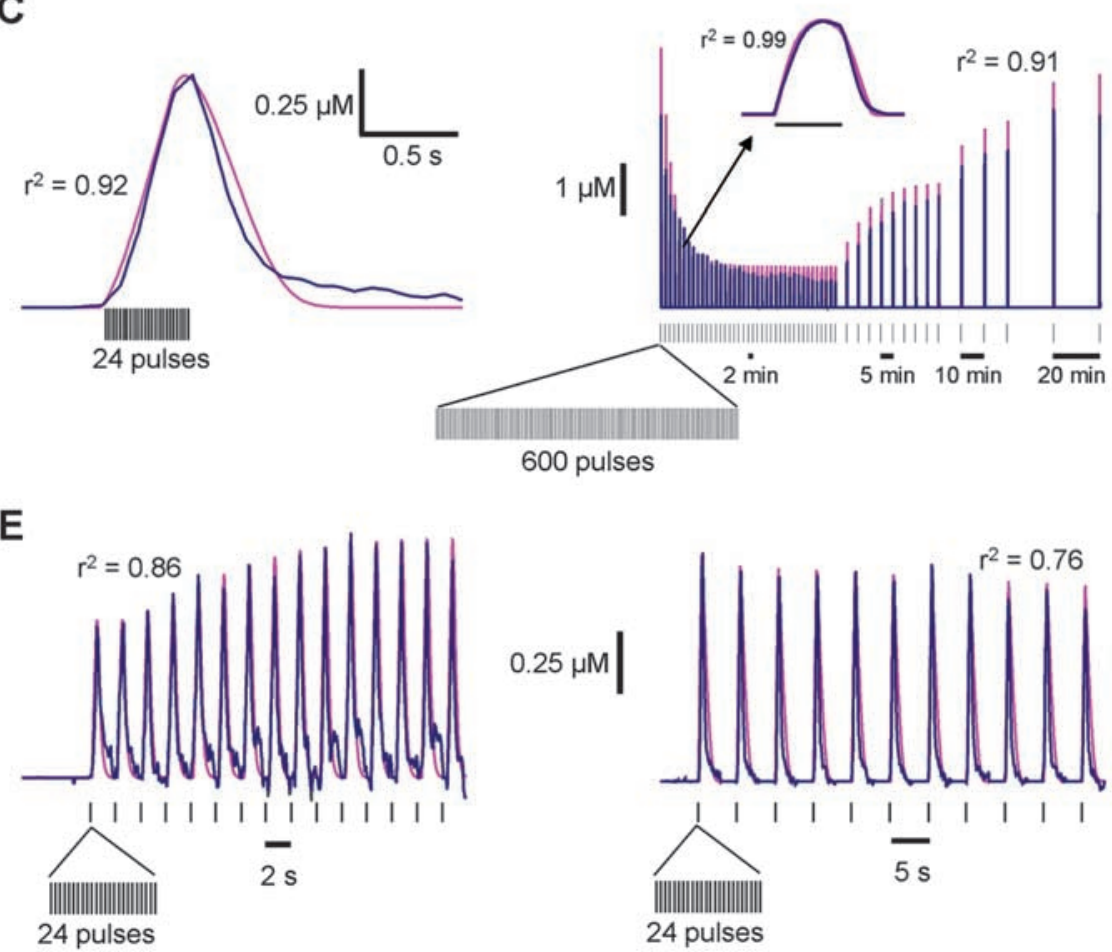

Figure 2. Dynamic model reveals multiple adaptive mechanisms for ongoing dopamine release. A, Semi-log plot of the moralized error for fitting the model to experimental data versus the number of independent dynamic components $l_{j}$. Representative fits are shown from four representative animals. The error decreases up to three components and does not improve appreciably beyond that. $B$, The three dynamic components consistently captured by the model: short-lasting facilitation (top), short-lasting depression (middle), and longer-lasting depression (bottom). These time-dependent mathematical components are induced by each action potential (arrow) and multiplicatively modify the amplitude of dopamine release for future action potentials. C, Fit of dynamic model to dopamine released during a single repetitive electrical stimulation ( 24 pulses, $60 \mathrm{~Hz}, 120 \mu \mathrm{A}$ ) applied to dopamine neurons of an ambulant rat. In all panels, the model is magenta, data are blue, and $r^{2}$ is the correlation coefficient of the goodness of fit. $D$, Fit of model to dopamine fluctuations evoked in an anesthetized animal by more intense stimulus trains. Each repetitive stimulus (vertical bar) consisted of 600 pulses $(60 \mathrm{~Hz}, 120 \mu \mathrm{A})$. The repetitive stimulus trains were delivered at 2,5, 10, or 20 min intervals. Depression is present that is still evident on the next stimulus with 2 min interstimulus intervals. At longer intervals there is greater recovery of release. Inset, Response for a single 600 pulse train $(60 \mathrm{~Hz}, 120 \mu \mathrm{A})$. E, Fit of model to dopamine fluctuations evoked by stimuli as in ( repeated at 2 sec intervals. Facilitation is apparent. $F$, Stimuli as in $C$ repeated at 5 sec intervals. A gradual depression is apparent.
$0.93 \pm 0.06)$ to the three-component model were also found to irregular stimulus patterns in awake animals (Table 1). To test the limits of the system, experiments were done in anesthetized rats that permitted the use of much longer stimulations (600 pulses, $60 \mathrm{~Hz}$ ) (Fig. 2D). Even under these circumstances, the model fits the changing amplitude of dopamine transients across the entire period of the experiment $(>3 \mathrm{hr})$, as well as the shape of the 
Table 1. Best-fit parameter values

\begin{tabular}{|c|c|c|c|c|c|c|c|}
\hline \multirow[b]{2}{*}{ Interburst interval } & \multirow[b]{2}{*}{$a_{0}(\mathrm{nм})$} & \multicolumn{2}{|l|}{ Facilitation } & \multicolumn{2}{|l|}{ Depression } & \multirow[b]{2}{*}{$r^{2}$} & \multirow[b]{2}{*}{$n$} \\
\hline & & $\tau_{1}(\mathrm{sec})$ & $\mathrm{k}_{1}$ & $\tau_{2}(\mathrm{sec})$ & $k_{2}$ & & \\
\hline 1 sec interval & 10 & 4.16 & 1.012 & 3.53 & 0.997 & 0.99 & 1 \\
\hline 2 sec interval & 20 & 4.41 & 1.001 & 3.24 & 0.989 & 0.94 & 1 \\
\hline 5 sec interval & 30 & 4.70 & 1.003 & 3.23 & 0.989 & 0.76 & $5^{a}$ \\
\hline 10 sec interval & 20 & 4.12 & 1.027 & 3.24 & 0.977 & 0.88 & $4^{a}$ \\
\hline Irregular & $20 \pm 2$ & $5.45 \pm 0.47$ & $1.010 \pm 0.001$ & $4.38 \pm 0.28$ & $0.984 \pm 0.008$ & $0.93 \pm 0.06$ & 6 \\
\hline Anesthetized & 60 & 4.41 & 1.003 & 3.23 & 0.965 & 0.91 & 1 \\
\hline
\end{tabular}

The parameter values for the fast facilitation and fast depression terms from a three-component model were evaluated by curve-fitting to the experimental data. For each paradigm listed, the parameters were determined independently. The first four rows show the values for fixed-interval stimulation patterns. The fifth row shows the mean \pm SEM for all of the stimulation patterns derived from the lever-press records of self-stimulating animals. The final row shows the values for an experiment in an anesthetized rat. The repetitive stimulus consisted of 24 pulses in all experiments except the anesthetized animal (final row) where it was 600 pulses. $r^{2}$ is the correlation coefficient of the fit, and $n$ is the number of animals used for each paradigm.

${ }^{a}$ The traces obtained at 5 and 10 sec intervals were averaged from different animals before curve fitting to improve signal quality without modifying the values of the fitted dynamic components.

individual responses to stimulation trains (Fig. $2 D$, inset). Furthermore, when the stimulation amplitude was increased from 120 to $300 \mu \mathrm{A}, a_{0}$ increased proportionately, but all of the other parameters were preserved (data not shown).

The robustness of the model, as suggested by the error analysis, is also supported by the way in which the fitted parameter values generalize across animals, patterns of stimulation, and with anesthesia. The values for the facilitation and the shorterterm depression are summarized in Table 1. The longer-term depression time constant, $\tau_{3}$, was found to have a lower limit of 10 min from the short experiments. Longer experiments in anesthetized animals further constrained this value to $\sim 14 \mathrm{~min}$, a result consistent with previous findings (Michael et al., 1987). The time constants extracted by the model were further verified by an alternative approximation (Appendix, see supplementary material, available at www.jneurosci.org) that also consistently found two depression terms (one short $\sim 3.5 \mathrm{sec}$; one long $\sim 12-15 \mathrm{~min}$ ) and one facilitation term (short $\sim 4.5 \mathrm{sec}$ ).

In contrast to the consistency of the time constants, the $k_{j}$ (kick) parameters were more variable between recording locations and animals (Table 1) as is $a_{0}$. Indeed, $a_{0}$ was used as an adjustable parameter because it varies with the density of release sites adjacent to the specific recording site (Wightman and Zimmerman, 1990). Despite this variability, the model may be used to predict dopamine fluctuations in response to novel stimulus patterns (Fig. 3). The $\tau_{\mathrm{j}}$ and $k_{\mathrm{j}}$ parameters extracted from a regularly repeated (every $1 \mathrm{sec}$ ) stimulation trains (Fig. $3 A$ ) were used to predict dopamine fluctuations for a stimulation pattern repeated at irregular time intervals in a different rat (Fig. 3B). Remarkably, the model accurately predicts the changes in extracellular dopamine concentration $\left(r^{2}=0.96\right)$, including the facilitation seen initially and the almost complete attenuation of release at the end of the train. The generalization across animals and stimulation patterns is evident in Figure $3 D$, in which the short-term facilitation and depression time constants are plotted against one another. There is a clear clustering in this plot. Here, there are six points that represent fits to irregular patterns of stimulation along with seven other points that represent regular patterns in awake and anesthetized animals. The points for the fits in Figure $3 A-C$ are shown as color-coded circles. The average for the entire ensemble of fits is shown with error bars extending along both axes.

\section{Discussion}

These results establish that ongoing dopamine release is controlled by adaptive mechanisms rather than having a fixed amplitude. These are analogous to the numerous mechanisms of gain control observed at glutamatergic synapses with postsynaptic recordings

(Markram et al., 1997; Bear, 1999; Malenka and Nicoll, 1999; Abbott and Nelson, 2000). Here we were able to avoid the confound of postsynaptic receptor changes while studying gain control of dopamine release (a presynaptic phenomenon) by using direct chemical measurements. The fluctuations of dopamine release could be described with a three-component dynamic model that robustly captured the short-term plasticity. The modeled facilitation and depression time scales were consistent across animals and stimulation patterns, and were not changed by anesthesia (Table 1, Figs. 2, 3).

\section{Physiological relevance of electrical stimulation}

In this study, dopamine release was evoked using electrical stimulation of dopamine cell bodies. The current used for most experiments $(120 \mu \mathrm{A})$ produces $\sim 30-50 \%$ of maximal striatal dopamine release (Wiedemann et al., 1992), suggestive of synchronous activation of $\sim 50 \%$ of dopamine neurons in the ipsilateral substantia nigra and ventral tegmental area. The level of synchronicity observed for behaviorally salient stimuli is at least this high (Hollerman and Schultz, 1998; Hyland et al., 2002). The number of spikes in a burst can be numerous in alert rats (up to 20; Freeman et al., 1985), and the instantaneous frequency can be high, especially in response to a rewarding stimulus $(27 \%$ of intraburst intervals have an instantaneous frequency of $>50 \mathrm{~Hz}$; Hyland et al., 2002). Although the dopamine increases were similar to that for a salient behavioral stimulus (Fig. 1A) (Robinson et al., 2002), the repetitive stimulation we used for most experiments (24 pulses, $60 \mathrm{~Hz}$ ) represents an extreme and almost certainly supraphysiological activation. Nonetheless, this type of stimulation is useful to expose the underlying physiological dynamics. Because the model, which describes events on a singleimpulse basis, generalizes well across different stimulation patterns and across experiments where different repetitive stimuli were used, it succeeds in capturing the physiology at its fundamental unit (a single action potential).

\section{Possible physiological mechanisms underlying the observed dynamics}

In the simplest scenario, each dynamic factor could be associated with an identifiable physiological process. Most experiments used submaximal stimulation current to avoid masking physiological effects at the cell body (e.g., hyperpolarization) that may effect action potential propagation. However, even with a supramaximal stimulation current, the dynamics of dopamine release were preserved, suggesting that their control resides exclusively in the terminal. The long-lasting depression is on the same time 
scale as that limited by the rate of dopamine biosynthesis and vesicular packaging (Michael et al., 1987). Inhibition of release through terminal autoreceptors decays with a time constant exactly in the range of the shorter-term depression (Phillips et al., 2002), and so this could be a major contributor to that component. However, it should be noted that shortlasting autoreceptor-independent depression has also been observed with (more promiscuous) local stimulation (Phillips et al., 2002; Cragg, 2003). Facilitation of dopamine may be a result of increased refilling of the readily releasable vesicular pool (Yavich and MacDonald, 2000), a function that is regulated by intracellular calcium (Wang and Kaczmarek, 1998). Indeed, calcium dynamics have been demonstrated to be an important factor that influences pulse-to-pulse dopamine release (Phillips and Stamford, 2000; Cragg, 2003). Changes in the rate of uptake could also modulate extracellular dopamine. However, the three component model based on plasticity of release captured the data remarkably well without alteration of uptake parameters. Future experiments are required to test these hypotheses and establish the precise control points of dopamine dynamics.

\section{Possible computational interpretation of dynamic filtering of dopaminergic spikes}

Extensive electrophysiological work in primates (Hollerman and Schultz, 1998; Schultz and Dickinson, 2000) has led to the theory that phasic activity of midbrain dopamine neurons encodes a prediction error signal in the estimation of future reward (Montague and Sejnowski, 1994; Montague et al., 1996; Schultz et al., 1997). Our findings show that the transformation of such spike activity (and presumably the prediction errors that they embody) into dopamine release adapts according to spike history. Through circuit-level adaptation, the average firing that encodes the prediction error is driven to zero, optimizing the prediction of the time and magnitude of rewarding events in the near future (Schultz et al., 1997). However, all real-world scenarios are associated with variability that no amount of learning can eliminate, that is, irreducible uncertainty. The longlasting depression component will eventually drive dopamine transmission to zero for sustained spiking (Fig. $1 B$, arrow 3 ), thus providing an additional level of filtering at the dopamine terminal. The functional relevance of the short-lasting facilitation and depression components are less clear. There is evidence that short-term dynamics are necessary to account for human performance on specific repeated-play decision-making tasks (such as in Montague and Berns, 2002). In particular, subjects demonstrate a short-term memory for previous actions (a form of eligibility trace) that follows similar dynamics to those characterized here for dopamine release (R. Bogacz, S. McClure, J. Cohen, and
R. Montague, personal communication). Based on these findings, it is tempting to speculate that the dynamic components that we have identified may be the physical substrate for the eligibility traces in reward-dependent decision tasks given to humans (Bogacz, McClure, Cohen, and Montague, personal communication). This is particularly provocative because dopamine release is strongly implicated in biasing action selection (Phillips et al., 2003b). Thus, dynamic adaptation of dopamine release has broad implications for multiple aspects of behavior.

\section{References}

Abbott LF, Nelson SB (2000) Synaptic plasticity: taming the beast. Nat Neurosci 3:1178-1183.

Abbott LF, Varela JA, Sen K, Nelson SB (1997) Synaptic depression and cortical gain control. Science 275:220-224.

Bath BD, Michael DJ, Trafton BJ, Joseph JD, Runnels PL, Wightman RM (2000) Subsecond adsorption and desorption of dopamine at carbonfiber microelectrodes. Anal Chem 72:5994-6002.

Bear MF (1999) Homosynaptic long-term depression: a mechanism for memory? Proc Natl Acad Sci USA 96:9457-9458.

Cragg SJ (2003) Variable dopamine release probability and short-term plas- 
ticity between functional domains of the primate striatum. J Neurosci 23:4378-4385.

Freeman AS, Meltzer LT, Bunney BS (1985) Firing properties of substantia nigra dopaminergic neurons in freely moving rats. Life Sci 36:1983-1994.

Garris PA, Christensen JRC, Rebec GV, Wightman RM (1997) Real-time measurement of electrically evoked extracellular dopamine in the striatum of freely moving rats. J Neurochem 68:152-161.

Garris PA, Kilpatrick M, Bunin MA, Michael D, Walker QD, Wightman RM (1999) Dissociation of dopamine release in the nucleus accumbens from intracranial self-stimulation. Nature 398:67-69.

Hollerman JR, Schultz W (1998) Dopamine neurons report an error in the temporal prediction of reward during learning. Nat Neurosci 1:304-309.

Hyland BI, Reynolds JN, Hay J, Perk CG, Miller R (2002) Firing modes of midbrain dopamine cells in the freely moving rat. Neuroscience 114:475-492.

Kilpatrick MR, Rooney MB, Michael DJ, Wightman RM (2000) Extracellular dopamine dynamics in rat caudate-putamen during experimenterdelivered and intracranial self-stimulation. Neuroscience 96:697-706.

Malenka RC, Nicoll RA (1999) Long-term potentiation-a decade of progress? Science 285:1870-1874.

Markram H, Lubke J, Frotscher M, Sakmann B (1997) Regulation of synaptic efficacy by coincidence of postsynaptic APs and EPSPs. Science 275:213-215

Montague PR, Berns GS (2002) Neural economics and the biological substrates of valuation. Neuron 36:265-284.

Michael AC, Ikeda M, Justice JB Jr (1987) Mechanisms contributing to the recovery of striatal releasable dopamine following MFB stimulation. Brain Res 421:325-335.

Montague PR, Sejnowski TJ (1994) The predictive brain: temporal coincidence and temporal order in synaptic learning mechanisms. Learn Mem $1: 1-33$.

Montague PR, Dayan P, Sejnowski TJ (1996) A framework for mesencephalic dopamine systems based on predictive Hebbian learning. J Neurosci 16:1936-1947.

Phillips PEM, Stamford JA (2000) Differential recruitment of N-, P- and Q-type voltage-operated calcium channels in striatal dopamine release evoked by "regular" and "burst" firing. Brain Res 884:139-146.

Phillips PEM, Hancock PJ, Stamford JA (2002) The time window of autoreceptor-mediated inhibition of limbic and striatal dopamine release. Synapse 44:15-22.

Phillips PEM, Robinson DL, Stuber GD, Carelli RM, Wightman RM (2003a) Real-time measurements of phasic changes in extracellular dopamine concentration in freely moving rats by fast-scan cyclic voltammetry. Methods Mol Med 79:443-464.
Phillips PEM, Stuber GD, Heien MLAV, Wightman RM, Carelli RM (2003b) Subsecond dopamine release promotes cocaine seeking. Nature 422:614-618.

Powell MJD (1964) An efficient method for finding the minimum of a function of several variables without calculating derivatives. Comput J 7:155-162.

Press WH, Flannery BP, Teukolsky SA, Vetterling WY (1990) Numerical recipes in Pascal, the art of scientific computing. Cambridge, UK: Cambridge UP.

Robinson DL, Heien MLAV, Wightman RM (2002) Frequency of dopamine concentration transients increases in dorsal and ventral striatum of male rats during introduction of conspecifics. J Neurosci 22:10477-10486.

Schultz W, Dickinson A (2000) Neuronal coding of prediction errors. Annu Rev Neurosci 23:473-500.

Schultz W, Dayan P, Montague PR (1997) A neural substrate of prediction and reward. Science 275:1593-1599.

Varela JA, Sen K, Gibson J, Fost J, Abbott LF, Nelson SB (1997) A quantitative description of short-term plasticity at excitatory synapses in layer $2 / 3$ of rat primary visual cortex. J Neurosci 17:7926-7940.

Venton BJ, Zhang H, Garris PA, Phillips PEM, Sulzer D, Wightman RM (2003) Real-time decoding of dopamine concentration changes in the caudate-putamen during tonic and phasic firing. J Neurochem 87:1284-1295.

Waelti P, Dickinson A, Schultz W (2001) Dopamine responses comply with basic assumptions of formal learning theory. Nature 412:43-48.

Wang LY, Kaczmarek LK (1998) High-frequency firing helps replenish the readily releasable pool of synaptic vesicles. Nature 394:384-388.

Wiedemann DJ, Garris PA, Near JA, Wightman RM (1992) Effect of chronic haloperidol treatment on stimulated synaptic overflow of dopamine in the rat striatum. J Pharmacol Exp Ther 261:574-579.

Wightman RM, Zimmerman JB (1990) Control of dopamine extracellular concentration in rat striatum by impulse flow and uptake. Brain Res Brain Res Rev 15:135-144.

Wightman RM, Amatore C, Engstrom RC, Hale PD, Kristensen EW, Kuhr WG, May LJ (1988) Real-time characterization of dopamine overflow and uptake in the rat striatum. Neuroscience 25:513-523.

Yavich L, MacDonald E (2000) Dopamine release from pharmacologically distinct storage pools in rat striatum following stimulation at frequency of neuronal bursting. Brain Res 870:73-79.

Yavich L, Tiihonen J (2000) In vivo voltammetry with removable carbon fibre electrodes in freely-moving mice: dopamine release during intracranial self-stimulation. J Neurosci Methods 104:55-63. 